\title{
Photobiomodulation for the Treatment of Primary Headache: Systematic Review of Randomized Clinical Trials
}

\author{
Andréa Oliver Gomes ${ }^{1}$, Ana Luiza Cabrera Martimbianco ${ }^{2}$, Aldo Brugnera Junior ${ }^{3}{ }^{\circ}$, \\ Anna Carolina Ratto Tempestini Horliana ${ }^{4}$ (D), Tamiris da Silva ${ }^{1}$, Elaine Marcílio Santos ${ }^{2}$, Yara Dadalti Fragoso ${ }^{2}$, \\ Kristianne Porta Santos Fernandes ${ }^{4}$, Samir Nammour ${ }^{5}\left[\right.$ and Sandra Kalil Bussadori ${ }^{1,4, *}$
}

check for updates

Citation: Gomes, A.O.; Martimbianco, A.L.C.; Brugnera Junior, A.; Horliana, A.C.R.T.; da Silva, T.; Santos, E.M.; Fragoso, Y.D.; Fernandes, K.P.S.; Nammour, S.; Bussadori, S.K. Photobiomodulation for the Treatment of Primary Headache: Systematic Review of Randomized Clinical Trials. Life 2022, 12, 98. https://doi.org/10.3390/ life12010098

Academic Editors

Massimiliano Valeriani and Jack Puymirat

Received: 3 December 2021

Accepted: 5 January 2022

Published: 11 January 2022

Publisher's Note: MDPI stays neutral with regard to jurisdictional claims in published maps and institutional affiliations.

Copyright: (C) 2022 by the authors. Licensee MDPI, Basel, Switzerland. This article is an open access article distributed under the terms and conditions of the Creative Commons Attribution (CC BY) license (https:// creativecommons.org/licenses/by/ $4.0 /)$.
1 Postgraduate Program in Rehabilitation Sciences, Universidade Nove de Julho (UNINOVE), Street Vergueiro, 235/249-Liberdade, São Paulo 05503-900, Brazil; an_oliver@hotmail.com (A.O.G.); tamiris.slv@hotmail.com (T.d.S.)

2 Postgraduate Program in Health and Environment, Universidade Metropolitana de Santos (UNIMES), São Paulo 05503-900, Brazil; analuizacabrera@hotmail.com (A.L.C.M.); elaine.marcilio@unimes.br (E.M.S.); yara.eegs@gmail.com (Y.D.F.)

3 Physics Institute of São Carlos (IFSC/USP), Universidade de São Paulo, São Paulo 05503-900, Brazil; aldo.brugnera@gmail.com

4 Postgraduate Program in Biophotonics Applied to Health Sciences, Universidade Nove de Julho (UNINOVE), São Paulo 05503-900, Brazil; annacrth@gmail.com (A.C.R.T.H.); kristianneporta@gmail.com (K.P.S.F.)

5 Department of Dental Science, Faculty of Medicine, University of Liege, 4000 Liege, Belgium; s.namour@uliege.be

* Correspondence: sandra.skb@gmail.com; Tel.: +55-11-3385924

Abstract: The purpose of this study was to evaluate the efficacy and safety of photobiomodulation as an adjuvant treatment for primary headache. A systematic review of randomized clinical trials was performed. For such, electronic searches were performed in the MEDLINE, Embase, Cochrane Library, LILACS, PEDro, PsycInfo, Clinicaltrials.gov., and WHO/ICTRP databases, with no restrictions imposed regarding language or year of publication. We included studies that assessed any photobiomodulation therapy as an adjuvant treatment for primary headache compared to sham treatment, no treatment, or another intervention. The methodological assessment was conducted using the Cochrane Risk of Bias tool. The certainty of the evidence was classified using the GRADE approach. Four randomized clinical trials were included. Most of the included studies had an overall high risk of bias. Compared to sham treatment, photobiomodulation had a clinically important effect on pain in individuals with primary headache. Despite the benefits reported for other outcomes, the estimates were imprecise, and the certainty of the evidence was graded as low. These findings are considered insufficient to support the use of photobiomodulation in the treatment of primary headache. Randomized clinical trials, with higher methodological quality, are needed to enhance the reliability of the estimated effects.

Keywords: pain; primary headache; photobiomodulation; low-level laser

\section{Introduction}

Headache is a common form of pain throughout the world. In most cases, the diagnosis of headaches is based entirely on patient history, as an isolated physical examination rarely provides adequate diagnostic information [1,2]. According to the World Health Organization, migraine is the second-highest leading cause of years lived with disability [3,4].

The International Headache Society published a classification system and operational diagnostic criteria for headache based on clinical consensus, classifying headache into primary (tension, migraine, or cluster) and secondary (e.g., caused by infection or vascular disease). The conventional treatment for primary headache is pharmacological (common analgesics or non-steroidal anti-inflammatory drugs). However, medication overuse can 
also be a cause of headache. In such cases, withdrawal should be counselled, so that the prophylactic treatment is effective [2].

Photobiomodulation with a low-level laser or light-emitting diode (LED) has been used for different therapeutic purposes since the end of the 1960s, when there was a considerable advance in the production of equipment and applications in the field of medicine [5-7]. Light at a wavelength in the red to infrared range of the spectrum (660 to $905 \mathrm{~nm}$ ) is generally employed because such wavelengths can penetrate the skin and deeper tissues, resulting in a reduction in inflammation, pain relief, and accelerated regeneration of tissues, or act as an acupuncture needle [8,9].

Photobiomodulation is a recent adjuvant treatment for numerous neurological and psychological conditions. It seems to increase the metabolic capacity of neurons, and stimulate anti-inflammatory, anti-apoptotic, and antioxidant responses as neurogenesis and synaptogenesis [10]. The noninvasive delivery of light to the head and brain through photobiomodulation therapy is commonly called transcranial PBMT. With this method, light passes through a set of layers, such as the scalp, periosteum, bone, meninges, and dura mater, and partially reaches the brain's cortical surface $[10,11]$.

Therefore, this systematic review was performed to evaluate the efficacy and safety of photobiomodulation as an adjuvant treatment for primary headache.

\section{Methods}

This systematic review followed the methodological recommendations of the Cochrane Handbook for Systematic Reviews of Interventions [12] and the PRISMA statement [13] to ensure the quality of the report. In addition, the review protocol was prospectively registered with the International Prospective Register of Systematic Reviews (PROSPERO) under CRD42021223429.

\subsection{Eligibility Criteria}

\subsubsection{Types of Included Studies}

We only considered randomized clinical trials (RCT) with parallel or crossover designs, independently of the publication status (complete article or abstract).

\subsubsection{Types of Participants}

We included RCTs involving adults (18 years of age or older) diagnosed with primary headache.

\subsubsection{Types of Interventions}

We included RCTs that assessed any photobiomodulation therapy as an adjuvant treatment for primary headache. The following comparators were considered: placebo (sham), no intervention, or different interventions, such as botulinum toxin type A, acupuncture, among others.

\subsubsection{Types of Outcome Measures}

Primary Outcomes

- Pain intensity during a headache episode, measured by any validated scale or questionnaire, such as visual analogue scale (VAS), numeric rating scale, among others.

- Severity, duration, and frequency of episodes of headache.

- Serious adverse events during or after treatment that could lead to hospitalization or death.

\section{Secondary Outcomes}

- Minor adverse events, measured as the proportion of participants with at least one adverse event during or after treatment (e.g., exacerbation of pain, discomfort).

- Analgesics needed.

- Quality of life (measured by valid questionnaires, such as the SF-36). 


\section{- $\quad$ Patient acceptability.}

The evaluation time of the outcomes was considered according to the follow-up analyzed in the included RCTs. In addition, the following intervals were considered for possible grouping in a meta-analysis: immediately after treatment, short term (up to three months after treatment), and long term (more than three months after treatment).

\subsection{Search Strategies}

Comprehensive and sensitive search strategies were conducted in the following six electronic databases on 14 February 2021, with no restrictions imposed regarding language or publication date: MEDLINE (via PubMed), EMBASE (via Elsevier), The Cochrane Central Register of Controlled Trials (CENTRAL) (via Wiley), Physiotherapy Evidence Database (PEDro), Literatura Latino Americana e do Caribeem Ciências da Saúde (LILACS (Latin American and Caribbean Health Sciences Literature) via Biblioteca Virtual emSaúde (Virtual Health Library)), and PsycInfo (via APA).

Ongoing clinical trials were searched in the following clinical trial registry databases: ClinicalTrials.gov (www.clinicaltrials.gov, accessed on 24 December 2021) and International Clinical Trials Registry Platform (ICTPR) of the World Health Organization (apps.who.int/ trialsearch). In addition, the grey literature was searched in the OpenGrey database (www.opengrey.eu, accessed on 24 December 2021). The search strategies are presented in Supplementary Table S1. Finally, hand searches were also performed in the reference lists of relevant studies.

\subsection{Study Selection and Data Extraction}

The references retrieved by the search strategies were selected by two authors, independently, through the Rayyan platform [14]. After removing the duplications, the authors analyzed the titles and abstracts, and studies that did not meet the eligibility criteria were excluded. In the second stage, studies with potential inclusion were analyzed in full text to decide whether to include or exclude. A third reviewer solved disagreements.

Two authors independently extracted the following information on included RCTs using a form previously prepared in Microsoft Office Excel ${ }^{\circledR}$ : sample size, characteristics of the participants (age, sex, and duration of pain), aspects of the intervention and comparator groups, analyzed outcomes, follow-up time and results. When necessary, the authors of the trials were contacted to provide additional information or test data.

\subsection{Methodological Quality Assessment of the Included Studies (Risk of Bias)}

The methodological quality of the included studies was assessed by two independent reviewers using the Cochrane Risk of Bias (RoB) table [12]. This tool comprises the following seven domains: random sequence generation, allocation concealment, blinding of participants and personnel, blinding of outcome assessors, incomplete outcome data, selective reporting and other sources of bias. Each included RCT was classified as having a low, unclear, or high risk of bias for each domain.

\subsection{Data Synthesis}

When possible (homogeneous studies and available data), meta-analyses were planned to be performed using the Review Manager 5.4.1 software (RevMan 5.4.1) with a random effects model. The measure of effect used for data on dichotomous outcomes was the relative risk (RR), and for continuous outcomes, the difference in means (DM), both with a $95 \%$ confidence interval (95\% CI). The heterogeneity between included studies was planned to be assessed by the $\mathrm{I}^{2}$ statistic, where greater than $50 \%$ is considered substantial heterogeneity [12]. However, in this systematic review, it was not possible to group data from included studies into a meta-analysis with considerable clinical variability between them. We also planned to conduct a publication bias analysis if more than ten studies were included in the meta-analysis. 


\subsection{Certainty of the Evidence}

The certainty of the body of evidence was appraised for each outcome using the Grading of Recommendations Assessment, Development, and Evaluation (GRADE) approach [15].

\section{Results}

\subsection{Search Results}

The search strategies retrieved 693 records. After removing 64 duplicates, 629 records were analyzed based on the title and abstract, and 620 were excluded for not meeting the inclusion criteria. Nine studies were analyzed by full text, and the following three were excluded: one for not performing the comparison of interest [16], one for not being an RCT, [17], and one for combining therapeutic interventions [18]. Two ongoing studies were identified (CTRI/2020/05/024968 and NCT02969642). After the entire selection process, four RCTs [19-22] were included in this systematic review. Figure 1 details the article selection process.
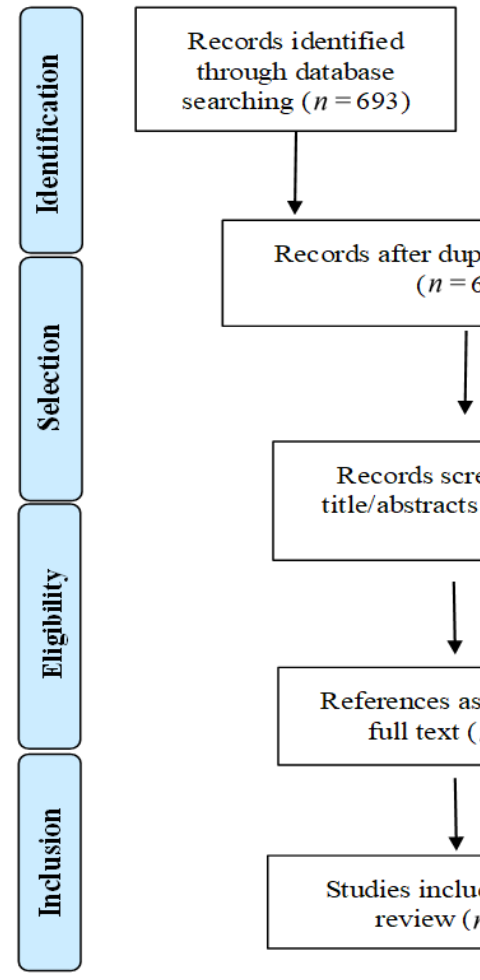

Additional records identified through other sources $(n=0)$
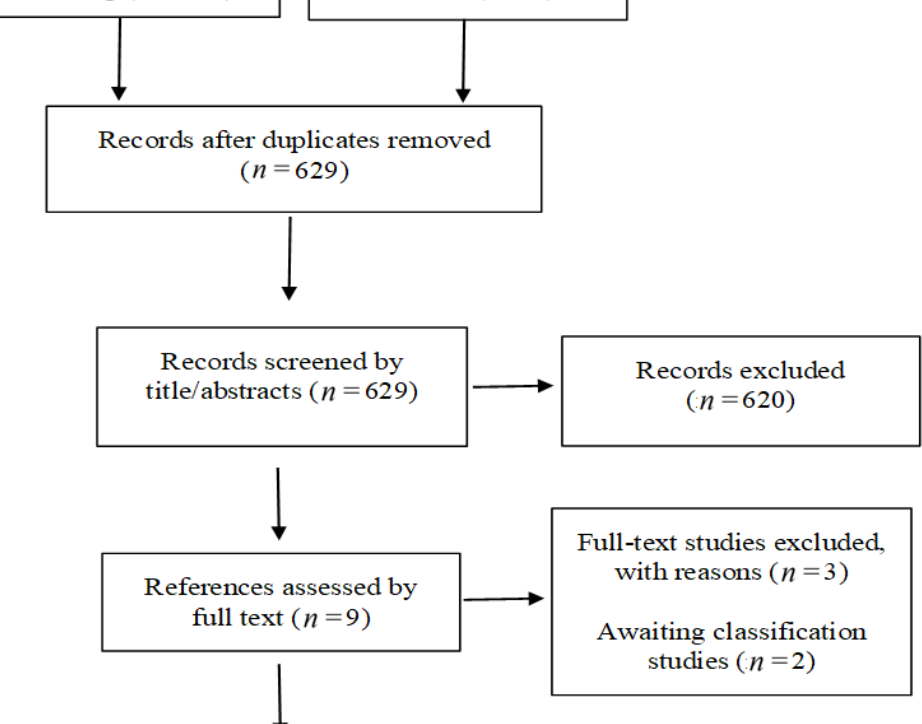

Studies included in the review $(n=4)$

Figure 1. PRISMA flow of the study selection process.

\subsection{Characteristics of Studies Included in the Review}

Table 1 displays the characteristics of the included RCTs. The studies were published between 1997 and 2020. One was published in Italy [19], one in Iran [20], one in Brazil [21], and one did not report the origin of the study [22]. The four RCTs involved 174 participants and evaluated photobiomodulation therapy to treat primary headache. The main inclusion criterion was the frequency of headache, with more than 15 days of pain per month. The main exclusion criteria were the overuse of analgesics and pre-existing conditions as other causes of headache.

All the included studies avoided the use of concomitant analgesics and prophylactic medication. 
Table 1. Main characteristics of the included studies.

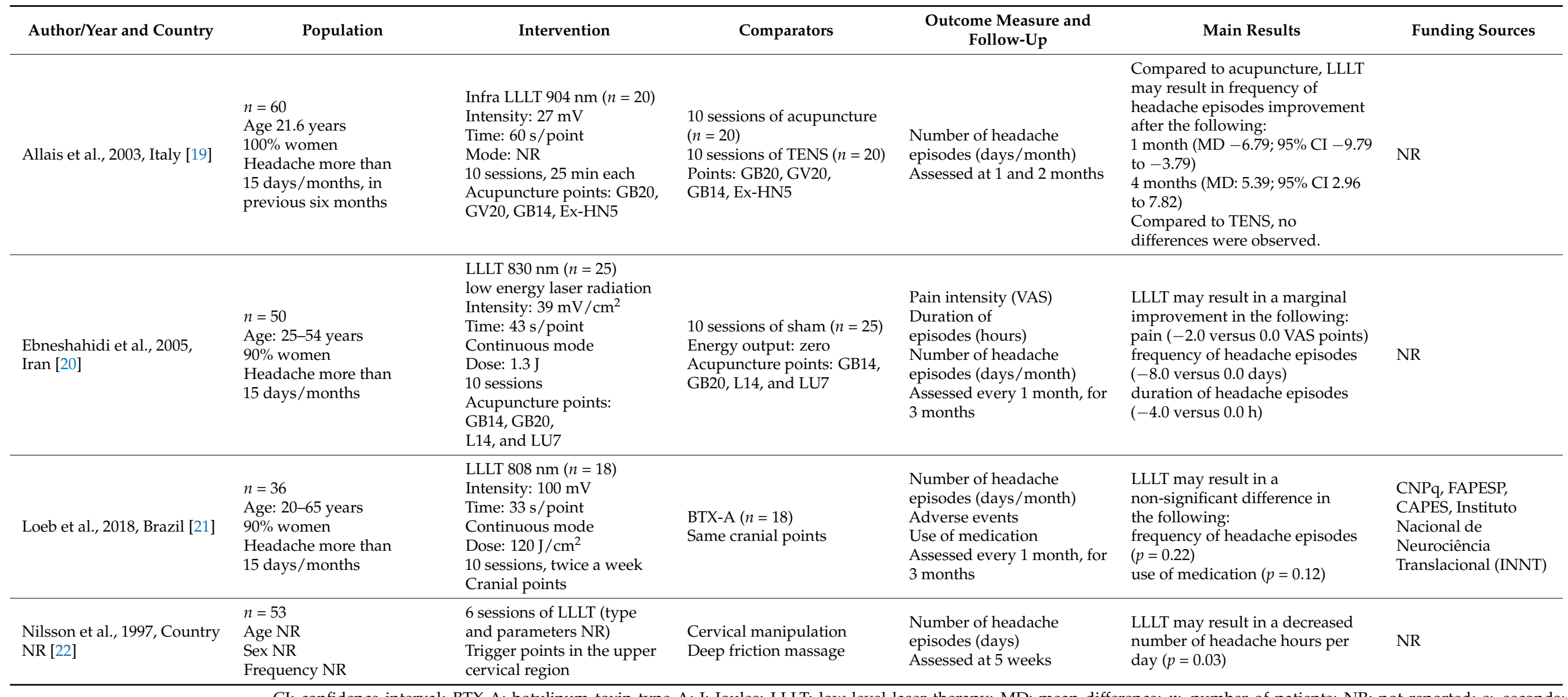

CI: confidence interval; BTX-A: botulinum toxin type A; J: Joules; LL
TENS: transcutaneous electrical nerve stimulation; VAS: visual analogue. 


\subsection{Methodological Quality Assessment of the Included Studies (Risk of Bias)}

Figure 2 summarizes the methodological quality assessment. Three of the four studies did not describe the randomization process or allocation concealment, and were classified as having an uncertain risk of bias for these domains. Three studies $[19,21,22]$ were judged to have a high risk of bias for blinding of the participants and therapists, due to the failure to provide sufficient information; as the interventions were different and the outcomes analyzed were subjective, it is likely that blinding was not performed, which could have influenced the final results. Similarly, two studies $[20,21]$ were judged to have a high risk of bias regarding the blinding of the assessors of the outcomes. None of the four studies reported having registered the protocol of the study. One study [22] was judged to have an uncertain risk of bias regarding "other sources of bias" due to the failure to describe the initial characteristics of the participants to enable a comparison of the groups at baseline.

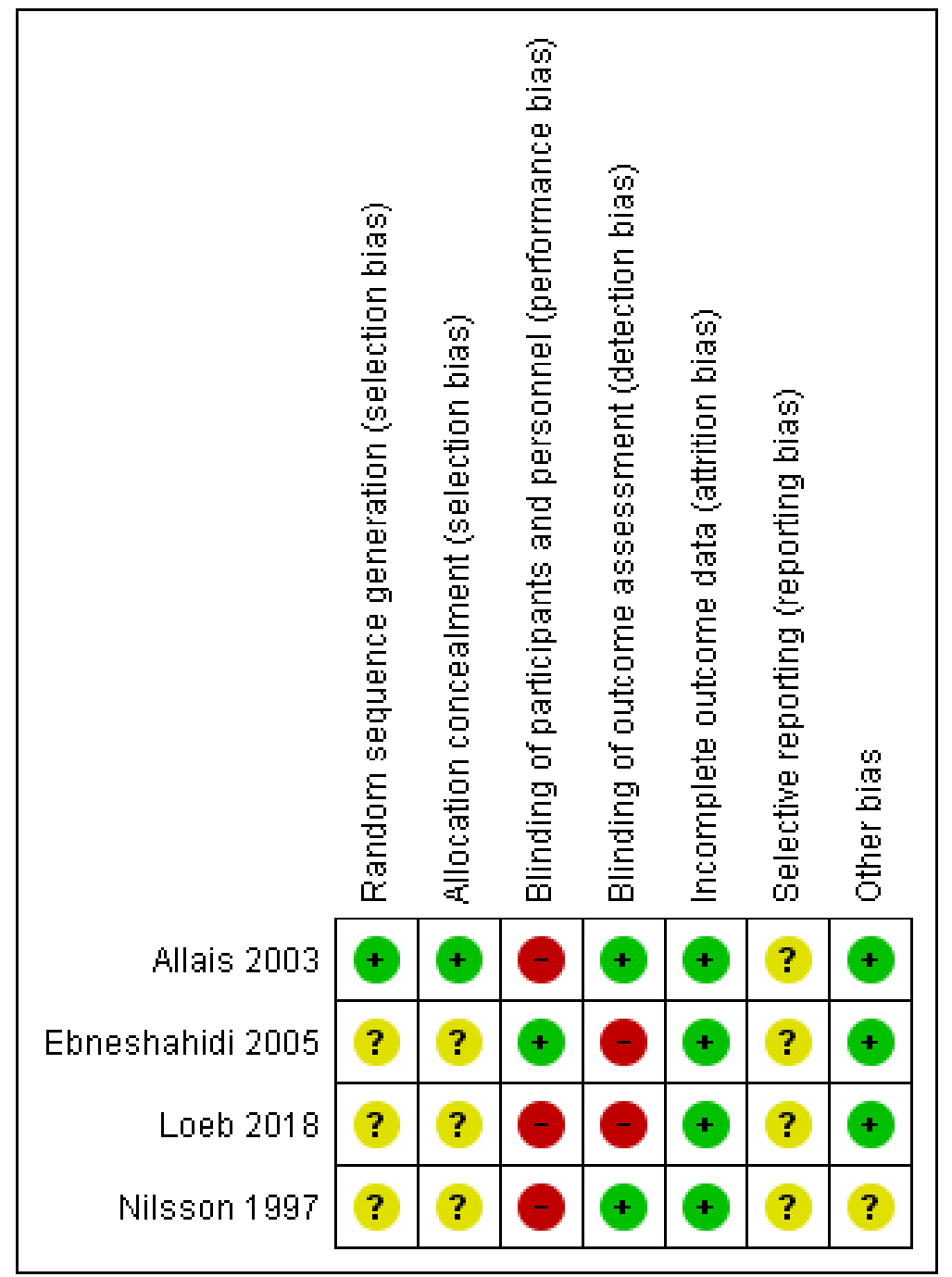

Figure 2. Risk of Bias assessment for each included study. $(+)=$ low risk of bias; (?) = unclear risk of bias; $(-)=$ high risk of bias.

\subsection{Effects of Intervention}

3.4.1. Comparison 1. Low-Level Laser Therapy (LLLT) versus Sham

A study with 50 participants [20] compared LLLT to sham (10 sessions over three weeks), and assessed the following outcomes after 2 months of treatment: 
- Improvement in pain: Pain was assessed using the visual analogue scale (VAS). An improvement in pain was found favouring the LLLT group (mean $-2.0 \pm 6.3$ versus $0 \pm 0 ; p<0.001)$.

- $\quad$ Frequency of headache episodes per day: an important reduction in headache episodes was found in the LLLT group compared to the sham group $(-8.0 \pm 21.5$ versus $0.0 \pm 0$; $p<0.001$ ).

- Duration of episodes (in hours): an improvement was found favoring the LLLT group $(-4.0 \pm 7.5$ versus $0.0 \pm 0.0 ; p<0.001)$.

\subsubsection{Comparison 2: LLLT versus Botulinum Toxin Type A (BTX-A)}

One study [21] compared the effects of LLLT to BTX-A, evaluating the following outcomes after 3 months of treatment:

- $\quad$ Frequency of headache episodes: no significant difference between groups was found regarding the reduction in episodes $(p=0.22)$.

- Adverse events: some patients in the LLLT group described a burning sensation at the application points, which disappeared at the end of the application.

- Use of analgesics: no significant difference between groups was found at the end of treatment $(p=0.12)$.

\subsubsection{Comparison 3. LLLT versus Acupuncture}

A study with 60 participants [9] compared the effect of infrared LLLT (ten 30 min sessions) to acupuncture (twice per week during the first week and weekly during the subsequent six weeks). The effect estimates showed a change in direction throughout the evaluation period of the study, as follows:

- Immediately after treatment: no significant difference in the frequency of episodes of headache (days/month) (mean difference (MD): 0.38; 95\% confidence interval [CI]: -2.43 to $3.19 ; p=0.79)$.

- One month after treatment: a significant reduction in the frequency of episodes was found in the LLLT group compared to the acupuncture group (MD -6.79; 95\% CI: -9.79 to $-3.79 ; p<0.00001)$.

- Four months after treatment: an increase in the number of episodes was found in the LLLT group, and a significant reduction was found in the acupuncture group (MD: $5.39 ; 95 \%$ CI: 2.96 to $7.82 ; p<0.00001)$.

The authors reported no adverse events stemming from the interventions during the study period.

\subsubsection{Comparison 4. LLLT versus Transcutaneous Electrical Nerve Stimulation (TENS)}

A study with 40 participants [19] compared the effect of infrared LLLT (ten 25 min sessions on alternate days) to TENS (ten $30 \mathrm{~min}$ daily sessions). No significant difference between groups was found regarding the reduction in the frequency of episodes of headache (days/month) at any of the evaluation times, as follows:

- Immediately after treatment: (MD: $-0.01 ; 95 \% \mathrm{CI}:-2.97$ to $2.95 ; p=0.99$ );

- One month after treatment (short term) (MD: 0.18; 95\% CI: -3.43 to 3.79; $p=0.92$ );

- Four months after treatment (long term) (MD: $-0.89 ; 95 \% \mathrm{CI}:-3.30$ to $1.52 ; p=0.47$ ).

The authors reported no adverse events stemming from the interventions during the study period.

None of the included studies assessed the quality of life and patient acceptability.

\subsection{Certainty of the Evidence}

The certainty of the body of evidence was classified as very low for all the outcomes analyzed in all the comparisons, due to the methodological limitations, small sample sizes, and imprecision. This indicated that we are uncertain about the effects of photobiomodulation for treating primary headache. 


\section{Discussion}

This systematic review assessed the effects of photobiomodulation as adjuvant therapy for primary headache. This intervention has been extensively investigated for several clinical conditions, given its possible effects on pain reduction, muscle relaxation, and tissue regeneration, consequently to metabolic, anti-inflammatory, and/or antioxidant responses $[10,11]$. The results of this systematic review showed no relevant difference between PBM therapy and the following interventions evaluated in the included studies: analgesic therapy using TENS, acupuncture, and botulinum toxin type A [19]. Some studies $[5,23,24]$ found that photobiomodulation was not superior to other therapies for the treatment of headaches, whereas another study [20] suggested improvements in pain and the frequency and duration of episodes of headache compared to sham treatment. In addition, no adverse events were reported, indicating this intervention's safety. Nevertheless, these findings should be interpreted with caution, given the low methodological quality of the available evidence until now. Other studies [15,19,22], with the same level of evidence, suggested that photobiomodulation was not superior to other manual therapies, such as cervical manipulation and deep friction massage. However, the use of analgesic medication was not reported, impeding an assessment of the real effect of these treatments. It is worth mentioning that none of the included studies assessed clinically relevant outcomes, such as quality of life and patient satisfaction. These outcomes could directly assess the patient report, regarding treatment and its impact on the routine.

Four RCTs [19-22] were included in the present systematic review, most of which had a high risk of bias. The certainty of the body of evidence was classified as very low, which indicates that there are uncertainties about the effects of photobiomodulation for primary headache, and more significant randomized clinical trials are required. Blinding of the participants, allocation concealment, and intention-to-treat analysis were rarely used. Moreover, flaws were detected that downgraded the certainty of the evidence and reduced the confidence in the results. The majority of the RCTs included in this review had a small sample size (average of 18 participants per group) and either failed to describe the calculation of the sample size or described it inadequately, likely due to the considerable heterogeneity in the photobiomodulation parameters (irradiance, quantity of energy delivered, and duration and frequency of the treatments), characteristics of the participants, selection of outcome measures, and methodological quality.

The choice of dosimetric parameters is crucial to the effectiveness of photobiomodulation. Energy quantities inside the recommended therapeutic window were recommended for many diseases [25]. Unfortunately, we have no ideal parameters for primary headache. Thus, the interpretation of the results requires an assessment of the adequacy of the dosimetric parameters for photobiomodulation [5].

Moreover, most trials in the present review reported these parameters inadequately or insufficiently. Thus, information on the dosimetric parameters provided in all the RCTs [19-22] was incomplete, which could lead to difficulty in reproducing the protocol and underscores the need to exercise caution when interpreting the results and conclusions of the present systematic review.

Considerable dosimetric variation was found among the four clinical trials; the total energy ranged from 6 to $12 \mathrm{~J}$ per point, the irradiation time ranged from 33 to $60 \mathrm{~s}$, and the average output power ranged from 27 to $100 \mathrm{~mW}$. Furthermore, these parameters were not described in all the studies. The studies also failed to report the source of the choice of dosimetric parameters (the study used as a guide), and the optimization of these parameters was not discussed.

One study [20], with 50 participants, evaluated laserpuncture compared to sham, regarding pain and the frequency and duration of episodes of pain, reporting improvements in all these outcomes.

In comparing the effects of LLLT and BTX-A on the frequency of episodes of headache, Luana Mazzacoratti Loeb, et al. reported that treatments had the same efficacy with regards to the "use of analgesic" outcome [21] . 
Another study [19] compared the effect of infrared LLLT to acupuncture. The effect estimates showed conflicting changes depending on the time point. No significant difference between groups was found immediately after treatment. In contrast, the laser group exhibited better results than the acupuncture group one month after treatment, and the acupuncture group exhibited better results than the laser group four months after treatment. Although acupuncture studies on primary headache are starting to be realized [23], there is no evidence of the effect of acupuncture on migraine without aura [24]. In addition, electroacupuncture is one of several effective treatments for migraine pain. However, they conclude that multicentre studies with large sample sizes and well-designed randomized controlled trials are needed [26].

In comparing infrared LLLT and TENS [19], no significant difference between the groups was found, regarding the frequency of episodes of headache at any of the evaluation times.

Besides the inherent differences of each study, regarding the population, dosimetric parameters, and groups studied, divergences were found regarding the behaviour of the treatments over time. Moreover, the results should be interpreted with caution, due to the low quality of evidence.

One study [19] reported an absence of adverse effects with both treatments (LLLT and acupuncture). Another study [24] reported that some patients in the LLLT group described a burning sensation at the application points, which disappeared at the end of the application, and another study failed to evaluate adverse effects [18].

The strong points of the present review are the use of a sensitive search strategy in six databases and hand searches of manuscript reference lists, with no restrictions imposed regarding the language or date of publication. Moreover, two independent reviewers screened the trials, data extraction, and appraisal of methodological quality, as recommended by the Cochrane Handbook for Systematic Reviews of Intervention, and the certainty of the evidence was carefully appraised using the GRADE approach [15].

Future studies need to be based on the Consolidated Standards of Reporting Trials (CONSORT statement) [27]. Moreover, all photobiomodulation variables should be described clearly and thoroughly, preferably in a table to facilitate the visualization of the dosimetric parameters. Finally, randomized clinical trials with higher methodological quality, an increased number of participants, and long-term follow-up are needed to support photobiomodulation as adjuvant therapy for primary headache.

\section{Conclusions}

The current evidence does not support the use of photobiomodulation to reduce pain and the frequency of episodes of primary headache. Further randomized clinical trials, with adequate sample sizes and methodological quality, are needed to investigate the efficacy and safety of photobiomodulation as an adjuvant treatment for primary headache.

Supplementary Materials: The following supporting information can be downloaded at: https: / / www.mdpi.com/article/10.3390/life12010098/s1, Table S1: Search strategies for each database.

Author Contributions: Conceptualization, A.O.G. and A.L.C.M.; Methodology, A.O.G., A.L.C.M. and A.B.J.; Software, A.B.J.; Validation, A.O.G., A.C.R.T.H. and T.d.S.; Formal analysis, A.L.C.M. and A.C.R.T.H.; investigation, A.O.G., S.K.B.; Resources, A.O.G., A.L.C.M., A.C.R.T.H. and T.d.S.; Data curation, Y.D.F. and K.P.S.F.; Writing-original draft preparation, A.O.G., A.C.R.T.H. and A.L.C.M.; Writing-review and editing, A.O.G., A.L.C.M. and Y.D.F.; Visualization, A.O.G., E.M.S., Y.D.F., S.K.B.; Supervision, A.O.G., S.N. and S.K.B.; Project administration, A.O.G., S.N. and S.K.B. All authors have read and agreed to the published version of the manuscript.

Funding: This research received no funding.

Institutional Review Board Statement: Not applicable.

Informed Consent Statement: This systematic review followed the methodological recommendations of the Cochrane Handbook for Systematic Reviews of Interventions and the PRISMA statement 
to ensure the quality of the report. In addition, the review protocol was prospectively registered with the International Prospective Register of Systematic Reviews (PROSPERO) under CRD42021223429.

Data Availability Statement: Not applicable.

Acknowledgments: Nove de Julho University for administrative and technical support.

Conflicts of Interest: All authors declare that there are no conflicts of interest.

\section{References}

1. Barry, L.H.; Matheson, E.M. Approach to Acute Headache in Adults. Am. Fam. Physician 2013, 87, 682-687.

2. Sheng, P. Headache Classification Subcommittee of the International Headache Society. The International Classification of Headache Disorders: 2nd edition. Cephalalgia 2004, 24 (Suppl. S1), 9-160.

3. James, S.L.; Abate, D.; Abate, K.H.; Abay, S.M.; Abbafati, C.; Abbasi, N. Global, regional, and national incidence, prevalence, and years lived with disability for 354 Diseases and Injuries for 195 countries and territories, 1990-2017: A systematic analysis for the Global Burden of Disease Study 2017. Lancet 2018, 392, 1789-1858. [CrossRef]

4. Lombard, L.; Farrar, M.; Ye, W.; Kim, Y.; Cotton, S.; Buchanan, A.S.; Jackson, J.; Joshi, S. A global real-world assessment of the impact on health-related quality of life and work productivity of migraine in patients with insufficient versus good response to triptan medication. J. Headache Pain 2020, 21, 41. [CrossRef]

5. $\quad$ Liebert, M.A.; Bjordal, J.A.N.M.; Johnson, M.I.; Iversen, V.; Lopes-Martins, R.A.B. Low-Level Laser Therapy in Acute Pain: A Systematic Review of Possible Mechanisms of Action and Clinical Effects in Randomized Placebo-Controlled Trials. Photomed. Laser Surg. 2006, 24, 158-168.

6. da Silva, J.P.; da Silva, M.A.; Almeida, A.P.F.; Lombardi Junior, I.; Matos, A.P. Laser therapy in the tissue repair process: A literature review. Photomed. Laser Surg. 2010, 28, 17-21. [CrossRef] [PubMed]

7. Luke, A.M.; Mathew, S.; Altawash, M.M.; Madan, B.M. Lasers: A review with their applications in oral medicine. J. Lasers Med. Sci. 2019, 10, 324-329. [CrossRef]

8. Cotler, H.B.; Chow, R.T.; Hamblin, M.R.; Carroll, J. The Use of Low Level Laser Therapy (LLLT) For Musculoskeletal Pain. MOJ Orthop. Rheumatol. 2015, 2, 68. [CrossRef]

9. Wang, X.; Tian, F.; Soni, S.S.; Gonzalez-Lima, F.; Liu, H. Interplay between up-regulation of cytochrome-c-oxidase and hemoglobin oxygenation induced by near-infrared laser. Sci. Rep. 2016, 6, 30540. [CrossRef]

10. Salehpour, F.; Mahmoudi, J.; Kamari, F.; Sadigh-eteghad, S.; Rasta, H.; Hamblin, M.R. Brain Photobiomodulation Therapy: A Narrative Review. Mol. Neurobiol. 2019, 55, 6601-6636. [CrossRef] [PubMed]

11. Hennessy, M.; Hamblin, M.R. Photobiomodulation and the brain: A new paradigm. J. Opt. 2017, 19, 13003. [CrossRef] [PubMed]

12. Higgins, J.P.T.; Thoma, J.; Chandler, J.; Cumpston, M.; Li, T.; Page, M.J.W.V. Cochrane Handbook for Systematic Reviews of Interventions, 2nd ed.; John Wiley \& Sons: Hoboken, NJ, USA, 2019.

13. Moher, D.; Liberati, A.; Tetzlaff, J.; Altman, D.G.; The PRISMA Group. Preferred reporting items for systematic reviews and meta-analyses: The PRISMA Statement. PLoS Med. 2009, 6, e1000097. [CrossRef]

14. Ouzzani, M.; Hammady, H.; Fedorowicz, Z.; Elmagarmid, A. Rayyan-a web and mobile app for systematic reviews. Syst. Rev. 2016, 5, 210. [CrossRef]

15. Guyatt, G.H.; Oxman, A.D.; Vist, G.E.; Kunz, R.; Falck-Ytter, Y.; Alonso-Coello, P.; Schünemann, H.J. GRADE: An emerging consensus on rating quality of evidence and strength of recommendations. BMJ 2008, 336, 924-926. [CrossRef] [PubMed]

16. Torelli, P.; Jensen, R.; Olesen, J. Physiotherapy for tension-type headache: A controlled study. Cephalalgia 2004, 24, 29-36. [CrossRef]

17. Amoils, S.; Krues, J. The effect of low level laser therapy on acute headache syndromes. Laser Ther. 1991, 3, 155-157. [CrossRef]

18. Lavies, N.G. Laser acupuncture for migraine and muscle tension headache: A double-blind controlled trial. Acupunct. Med. 1998, 16, 73-76. [CrossRef]

19. Allais, G.; De Lorenzo, C.; Quirico, P.; Lupi, G.; Airola, G.; Mana, O.; Benedetto, C. Non-pharmacological approaches to chronic headaches: Transcutaneous electrical nerve stimulation, lasertherapy and acupuncture in transformed migraine treatment. Neurol. Sci. 2003, 24 (Suppl. S2), s138-s142. [CrossRef]

20. Ebneshahidi, N.S.; Heshmatipour, M.; Moghaddami, A.; Eghtesadi-Araghi, P. The effects of laser acupuncture on chronic tension headache-A randomised controlled trial. Acupunct. Med. 2005, 23, 13-18. [CrossRef]

21. Loeb, L.M.; Amorim, R.P.; Mazzacoratti, M.d.G.N.; Scorza, F.A.; Peres, M.F.P. Botulinum toxin A (BT-A) versus low-level laser therapy (LLLT) in chronic migraine treatment: A comparison TT-Toxina botulínica A (BT-A) versus laser terapia de baixa potência (LLLT) em enxaqueca crônica: Uma triagem comparativa. Arq. Neuropsiquiatr. 2018, 76, 663-667. Available online: http:/ / www.scielo.br/scielo.php?script=sci_arttext\&pid=S0004-282X2018001000663 (accessed on 24 December 2021). [CrossRef]

22. Nilsson, N.; Christensen, H.W.H.J. The effect of spinal manipulation in the treatment of cervicogenic headache. J. Manip. Physiol. Ther. 1997, 20, 326-330.

23. Xu, S.; Yu, L.; Luo, X.; Wang, M.; Chen, G.; Zhang, Q.; Liu, W.; Zhou, Z.; Song, J.; Jing, H.; et al. Manual acupuncture versus sham acupuncture and usual care for prophylaxis of episodic migraine without aura: Multicentre, randomised clinical trial. BMJ 2020, 368, m697. [CrossRef] [PubMed] 
24. Li, X.; Dai, Q.; Shi, Z.; Chen, H.; Hu, Y.; Wang, X.; Zhang, X.; Tian, G. Clinical Efficacy and Safety of Electroacupuncture in Migraine Treatment: A Systematic Review and Network Meta-Analysis. Am. J. Chin. Med. 2019, 47, 1755-1780. [CrossRef]

25. World Association for Laser Therapy (WALT). Dosage Recommendations. Available online: https://waltza.co.za (accessed on 24 February 2021).

26. Xu, J.; Zhang, F.-Q.; Pei, J.; Ji, J. Acupuncture for migraine without aura: A systematic review and meta-analysis. J. Integr. Med. 2018, 16, 312-321. [CrossRef] [PubMed]

27. Schulz, K.F.; Altman, D.G.; Moher, D. Consort 2010 statement: Updated guidelines for reporting parallel group randomised trials. Int. J. Surg. 2011, 9, 672-677. [CrossRef] [PubMed] 